\title{
THE IDENTICAL EQUATIONS OF THE MULTIPLICATIVE FUNCTION
}

BY R. VAIDYANATHASWAMY

1. Introduction. An arithmetic function $f(N)$ is multiplicative, if $f(M N)=f(M) f(N)$, whenever the integers $M, N$ are relatively prime. An analogous definition may be given for functions of more than one argument; thus $\psi\left(M_{1}, M_{2}\right)$ would be said to be multiplicative, if $\psi\left(M_{1} N_{1}, M_{2} N_{2}\right)=\psi\left(M_{1}, M_{2}\right) \psi\left(N_{1}, N_{2}\right)$, whenever the two products $M_{1} M_{2}, N_{1} N_{2}$ are relatively prime. If $f(N)$ and $\psi\left(M_{1}, M_{2}\right)$ are multiplicative functions, it is clear that we have

$$
f(1)=\psi(1,1)=1 \text {. }
$$

If the relation $f(M N)=f(M) f(N)$ is true for all integers $M, N$, we shall say that $f$ is a linear function.

The process of composing two arithmetic functions $f(N), \phi(N)$ consists in forming their composite (represented by $f \cdot \phi$ ), namely the function $\psi$ defined by the equation

$$
\psi(N)=\sum f(\delta) \phi(N / \delta),
$$

where the summation is for all divisors $\delta$ of $N$. If $f$ and $\phi$ are multiplicative, it is easy to see that their composite $\psi$ is also multiplicative. The notion of composition may be obviously extended to functions of several arguments.

Given an arithmetic function $f(N)$, such that $f(1) \neq 0$, it has been shown by E. T. Bell, * that there exists a unique arithmetic function $\psi(N)$, such that the composite $f \cdot \psi$ vanishes for all values of its argument other than 1 , and takes the value 1 when the argument is equal to 1 . We call $\psi$ the inverse function of $f$, and denote it by $f^{-1}$; it is easy to see that $f^{-1}$ is multiplicative if $f$ is also.

We shall say that a multiplicative function $F\left(M_{1}, M_{2}\right)$ is a cardinal function of $M_{1}, M_{2}$, if it vanishes whenever either of

* On a certain inversion in the theory of numbers, Tôhoku Mathematical Journal, vol. 17 (1920). Also A ray of numerical functions, this Bulletin, vol. 32 (1926), p. 341. 
$M_{1}, M_{2}$ admits a divisor relatively prime to the other, in other words, whenever the distinct prime factors of $M_{1}$ are not identical with those of $M_{2}$. We require in what follows, a particular cardinal function $C\left(M_{1}, M_{2}\right)$ defined as follows:

$C\left(M_{1}, M_{2}\right)=0$, if the distinct prime factors of $M_{1}, M_{2}$ are not identical,

$C\left(M_{1}, M_{2}\right)=(-1)^{\nu}$, if $M_{1}, M_{2}$ have the same $\nu$ distinct prime factors.

I have shown elsewhere,* by the method of generating series, that every multiplicative function $f$ (of a single argument) satisfies a certain identical equation. By using the cardinal function $C$, the identical equation can be put into the form

$$
f(M N)=\sum f\left(\frac{M}{\delta_{1}}\right) f\left(\frac{N}{\delta_{2}}\right) f^{-1}\left(\delta_{1} \delta_{2}\right) C\left(\delta_{1}, \delta_{2}\right),
$$

the summation on the right extending over all divisors $\delta_{1}$ of $M$, and $\delta_{2}$ of $N$. My purpose in the present note is to show that this identical equation can be derived, by straightforward reasoning of a purely arithmetical nature, from certain fundamental properties of the inverse function.

2. Three Theorems relating to the Inverse Function.

THEOREM 1. If $f$ is any multiplicative function of one argument, and $f^{-1}$ its inverse function, then the sum

$$
\sum f\left(M_{1} \delta\right) f^{-1}\left(\frac{M_{2} N}{\delta}\right),
$$

extended over all the divisors $\delta$ of $N$, vanishes unless every prime factor of $N$ divides $M_{1} M_{2}$.

Proof. Let $N=N_{1} N_{2}$, where all the prime factors of $N_{1}$ divide $M_{1} M_{2}$, and $N_{2}$ is relatively prime to $M_{1} M_{2}$. It is clear that $N_{1}$ and $N_{2}$ are relatively prime, and therefore any factor $\delta$ of $N$ can be expressed uniquely in the form $\delta_{1} \delta_{2}$, where $\delta_{1}$ is a divisor of $N_{1}$, and $\delta_{2}$ of $N_{2}$. Hence we have

$$
\sum f\left(M_{1} \delta\right) f^{-1}\left(\frac{M_{2} N}{\delta}\right)=\sum f\left(M_{1} \delta_{1} \delta_{2}\right) f^{-1}\left(\frac{M_{2} N_{1}}{\delta_{1}} \cdot \frac{N_{2}}{\delta_{2}}\right)
$$

* In a paper on The theory of multiplicative arithmetic functions, to appear shortly in the Transactions of this Society. 


$$
\begin{aligned}
& =\left\{\sum f\left(\delta_{2}\right) f^{-1}\left(\frac{N_{2}}{\delta_{2}}\right)\right\}\left\{\sum f\left(M_{1} \delta_{1}\right) f^{-1}\left(\frac{M_{2} N_{1}}{\delta_{1}}\right)\right\} \\
& =0, \text { if } N_{2} \neq 1,
\end{aligned}
$$

which proves the theorem.

Corollary. A factor $N_{1}$ of $N$ may be called a block factor, if it is relatively prime to $N / N_{1}$. The sum

$$
\sum f\left(\frac{N}{\delta}\right) f^{-1}(\delta)
$$

extended over all the divisors $\delta$ of a block factor $N_{1}(\neq 1)$ of $N$, vanishes. This may be proved directly or as a consequence of the theorem. The defining property of the inverse function is the particular case of this result which arises when the block factor $N_{1}$ coincides with $N$.

Suppose now that $N$ contains $\nu$ distinct prime factors, and $N_{i}{ }^{1}, N_{i}{ }^{2}, \cdots, N_{i}^{k}\left(k=\left(\begin{array}{l}\nu \\ i\end{array}\right)\right)$ are the distinct block factors of $N$ which contain exactly $i$ of the prime factors. Consider the sum

$$
\begin{aligned}
& A=\sum_{N} f\left(\frac{N}{\delta}\right) f^{-1}(\delta)-\sum_{k=1}^{\nu}\left\{\sum_{N^{k}} f\left(\frac{N}{\delta}\right) f^{-1}(\delta)\right\} \\
&+\sum_{k=1}^{\nu(\nu-1) / 2}\left\{\sum_{\substack{N^{k} \\
\nu-1}} f\left(\frac{N}{\delta}\right)^{\left.f^{-1}(\delta)\right\}-\cdots}\right. \\
&+(-1)^{\nu-1} \sum_{k=1}^{\nu}\left\{\sum_{N_{1} k} f\left(\frac{N}{\delta}\right) f^{-1}(\delta)\right\},
\end{aligned}
$$

where $N_{i}^{j}$ below $\sum$ indicates that the sum is extended over all the divisors $\delta$ of $N_{i}^{j}$. We shall evaluate the expression $A$ in two ways. In the first place, every partial sum in $A$, except the first, vanishes by the Corollary to Theorem I. Hence we have

$$
A=\sum_{N} f\left(\frac{N}{\delta}\right) f^{-1}(\delta)=0, \quad(N>1) .
$$

On the other hand consider a particular divisor $d$ of $N$, containing $i$ distinct prime factors. The coefficient of $f(N / d) f^{-1}(d)$ in $A$ is

$$
1-\left(\begin{array}{c}
\nu-i \\
1
\end{array}\right)+\left(\begin{array}{c}
\nu-i \\
2
\end{array}\right)-\cdots=0
$$


if $0<i<\nu$, but $=1$ if $i=\nu$. If $d=1$, the coefficient of

is

$$
f(N / 1) f^{-1}(1)=f(N)
$$

$$
1-\left(\begin{array}{l}
\nu \\
1
\end{array}\right)+\left(\begin{array}{l}
\nu \\
2
\end{array}\right)-\cdots+(-1)^{\nu+1}\left(\begin{array}{c}
\nu \\
\nu-1
\end{array}\right)=(-1)^{\nu-1}
$$

hence we have

$$
A=\sum f\left(\frac{N}{t}\right) f^{-1}(t)+(-1)^{\nu-1} f(N)
$$

where the summation is for the divisors $t$ of $N$ which contain all its distinct prime factors. Since it has been shown that $A=0$, we have the following theorem.

THEOREM 2. If $N$ contains $\nu$ distinct prime factors, then

$$
\sum f\left(\frac{N}{t}\right) f^{-1}(t)=(-1)^{v} f(N),
$$

where the summation extends over all the divisors $t$ of $N$ which contain all its $\nu$ distinct prime factors.

The next theorem is concerned with two numbers $M, N$ which contain the same $\nu$ distinct prime factors. We shall denote by $M_{i}^{k}, N_{i}^{k}\left(k=1,2, \cdots,\left(\begin{array}{l}\nu \\ i\end{array}\right)\right)$ two block factors of $M, N$ respectively, which contain the same $i$ prime factors. We shall also write

$$
M=M_{i}^{k} \bar{M}_{i}^{k}, \quad N=N_{i}^{k} \bar{N}_{i}^{k},
$$

so that $\bar{M}_{i}{ }^{k}, \bar{N}_{i}{ }^{k}$ are respectively prime to $M_{i}{ }^{k}, N_{i}{ }^{k}$ and are block factors of $M, N$, containing the same $\nu-i$ prime factors. Consider the expression

$$
\begin{aligned}
B=\sum f & \left(\frac{M N}{\delta}\right) f^{-1}(\delta) \\
& +\sum_{k=1}^{\nu}\left\{\sum \sum f\left(\frac{M_{1}^{k}}{t} \cdot \frac{\bar{M}_{1}^{k} \bar{N}_{1}^{k}}{\delta}\right) f^{-1}\left(N_{1}^{k} t \delta\right)\right\} \\
& -\sum_{k=1}^{\nu(\nu-1) / 2}\left\{\sum \sum f\left(\frac{M_{2}^{k}}{t} \cdot \frac{\bar{M}_{2}^{k} \bar{N}_{2}^{k}}{\delta}\right) f^{-1}\left(N_{2}^{k} t \delta\right)\right\}+\cdots \\
& -\cdots+(-1)^{\nu-1} \sum f\left(\frac{M}{t}\right) f^{-1}(N t) .
\end{aligned}
$$


Here the first term of $B$ is a summation over all divisors $\delta$ of $N$. The second (and later) terms contain three summations; the two inner summations relate respectively to all divisors $\delta$ of $\bar{M}_{i}^{k} \bar{N}_{i}^{k}$, and to all such divisors $t$ of $M_{i}{ }^{k}$ as contain all its distinct prime factors; the outer summation relates to all possible resolutions of $M, N$ into corresponding block factors containing $i$ and $\nu-i$ primes. The signs of the $\nu+1$ terms in $B$ alternate from the second term onwards. In the last term $i=\nu$, and so the outer summation as well as the summation relating to $\delta$, has disappeared, leaving only the summation over all factors $t$ of $M$ containing all its $\nu$ prime factors.

As in the proof of Theorem 2, we shall evaluate $B$ in two different ways. In the first place all terms of $B$, except the first and the last, vanish. To see this, take a fixed divisor $t$ of $M_{i}{ }^{k}$ (containing all its prime factors), and consider the sum

$$
\sum f\left(\frac{M_{i}^{k}}{t} \cdot \frac{\bar{M}_{i}^{k} \bar{N}_{i}^{k}}{\delta}\right) f^{-1}\left(N_{i}^{k} t \delta\right)
$$

extended over all the divisors $\delta$ of $\bar{M}_{i}{ }_{i} \bar{N}_{i}{ }^{k}$. Since $\bar{M}_{i}^{k} \bar{N}_{i}^{k}$ has no prime factors in common with $M_{i}{ }^{k} / t \times N_{i}{ }^{k} t=M_{i}{ }^{k} N_{i}{ }^{k}$, it follows from Theorem 1 that this (and similarly every other sum of the same type) vanishes. It thus follows that

$$
B=\sum f\left(\frac{M N}{\delta}\right) f^{-1}(\delta)+(-1)^{\nu-1} \sum f\left(\frac{M}{t}\right) f(N t),
$$

where the first summation is for all divisors $\delta$ of $N$, and the second for all divisors $t$ of $M$ which contain all its $\nu$ distinct prime factors.

Secondly, we shall show that $B$ is equal to the sum $\sum f(M N / \delta) f^{-1}(\delta)$, extended over all divisors $\delta$ of $M N$, and is therefore identically zero. A divisor $d$ of $M N$ may divide $N$; if it does not divide $N$, then a certain number, say $i(\geqq 1)$, of the $\nu$ prime factors of $N$ must occur in $d$ to a higher power than in $N$. Denote by $N_{i}^{k}$ the block factor of $N$ corresponding to these $i$ primes; then we can express $d$ in the form

$$
d=N_{i}^{k} t \delta
$$

where $\delta$ is an arbitrary factor of $\bar{M}_{\imath}{ }^{k} \bar{N}_{\imath}{ }^{k}$, and $t$ is a factor of $M_{i}{ }^{k}$ containing all its prime factors $\left(M_{\imath}{ }^{k}, \bar{M}_{\imath}{ }^{k}, \bar{N}_{\imath}{ }^{k}\right.$ having the mean- 
ings already explained). But this expression for $d$ is not unique, since there is no restriction on the factor $\delta$ of $\bar{M}_{\imath}{ }^{k} \bar{N}_{\imath}{ }^{k}$; in fact, if $N_{j}^{l}(1 \leqq j<i)$ is a block factor of $N$, which divides $N_{i}^{k}$, we can also write

$$
d=N_{j}^{l} t_{1} \delta_{1}
$$

where $t_{1}$ is a divisor of $M_{j}^{l}$ containing all its prime factors, and $\delta_{1}$ is some divisor of $\bar{M}_{j} l \bar{N}_{j}^{l}$.

Consider now the number of times which $f(M N / d) f^{-1}(d)$ ( $d$ being a particular divisor of $M N$ ) occurs in $(B)$. In case $d$ divides $N$, it is clear that it occurs only once, namely in the first term of $B$. If $d$ does not divide $N$, let exactly $i$ of the $\nu$ prime factors occur in $d$ to a higher power than in $N$; from the previous explanation, it will be clear then that $f(M N / d) f^{-1}(d)$ will occur $\left(\begin{array}{l}i \\ 1\end{array}\right)$ times in the second term of $B$, and generally $\left(\begin{array}{l}i \\ \lambda\end{array}\right)$ times in the $(\lambda+1)$ th term. Hence its coefficient in $B$ is

$$
\left(\begin{array}{l}
i \\
1
\end{array}\right)-\left(\begin{array}{l}
i \\
2
\end{array}\right)+\cdots=1-(1-1)^{i}=1 \text {, if } i>0 \text {. }
$$

Thus every term $f(M N / d) f^{-1}(d)$ occurs just once in $B$, whether $d$ divides $N$ or not. Hence

$$
B=\sum_{\delta \mid M N} f\left(\frac{M N}{\delta}\right) f^{-1}(\delta)=0 \quad(\text { since } M N>1) .
$$

Combining this with our previous evaluation of $B$, we have the following result.

THEOREM 3. If $M$ and $N$ have the same $\nu$ distinct prime factors, then

$$
\sum f\left(\frac{M N}{\delta}\right) f^{-1}(\delta)=(-1)^{\nu} \sum f\left(\frac{M}{t}\right) f^{-1}(N t),
$$

where on the left the summation is for all divisors $\delta$ of $N$, and on the right for all divisors $t$ of $M$ which contain all its $\nu$ distinct prime factors.

Corollary 1. On putting $N=1$ in Theorem 3, we obtain Theorem 2. Hence the theorem must be considered to be true for any $M$, and $N=1$.

Corollary 2. Let $M_{1}$ be relatively prime to $M$ (and therefore 
also to $N$ ). Write $M^{\prime}=M_{1} M$, and multiply both sides of the equality in Theorem 3 by $f\left(M_{1}\right)$. We obtain the relation

$$
\sum f\left(\frac{M^{\prime} N}{\delta}\right) f^{-1}(\delta)=(-1)^{\nu} \sum f\left(\frac{M^{\prime}}{t}\right) f^{-1}(N t),
$$

where $M^{\prime}$ is any number containing all the $\nu$ distinct prime factors of $N$, the summation on the left is for all divisors $\delta$ of $N$, and on the right for all divisors $t$ of $M^{\prime}$ which contain only the $\nu$ distinct prime factors of $N$.

Corollary 3. Theorem 3 is also true for any two numbers $M, N$, provided (1) $\nu$ is the number of distinct prime factors in $N$, and (2) the summation on the right is for all divisors $t$ of $M$ which contain the $\nu$ distinct primes dividing $N$, and no others.

If all the $\nu$ prime factors of $N$ occur in $M$, then Corollary 3 reduces to Corollary 2. If on the other hand, some of the $\nu$ prime factors of $N$ do not occur in $M$, then the left side of the equality of Theorem 3 vanishes by virtue of Theorem I, and the right side also vanishes, since there are now no factors $t$ of $M$ which contain all the prime factors of $N$. Thus, under this interpretation of the summation on the right, Theorem 3 is true for any two numbers $M, N$.

3. The Identical Equation of $f$. Replacing $N$ by $N_{1}$, the equality of Theorem 3 is

$$
\sum f\left(\frac{M N_{1}}{\delta}\right) f^{-1}(\delta)=(-1)^{\nu} \sum f\left(\frac{M}{t}\right) f^{-1}\left(N_{1} t\right),
$$

where $\nu$ is the number of prime factors in $N_{1}, \delta$ runs over the divisors of $N_{1}$, and $t$ over those divisors of $M$ which contain neither more nor less than the $\nu$ prime factors of $N_{1}$. As has been remarked, this result is true for any two numbers $M, N_{1}$.

Multiply both sides of the equality by $f\left(N_{2}\right)$ and sum over all values of $N_{1}, N_{2}$, such that $N_{1} N_{2}=N$. On the left side, we carry out the summation in two stages; namely, we first keep $N_{1} / \delta$ fixed, and sum over all values of $N_{2}$ and $\delta$ such that $N_{2} \delta=N \delta / N_{1}$. Thus the left side is

$$
\sum \sum f\left(\frac{M N_{1}}{\delta}\right) f^{-1}(\delta) f\left(N_{2}\right)=\sum f\left(\frac{M N_{1}}{\delta}\right) \sum f^{-1}(\delta) f\left(N_{2}\right) .
$$


We have also

$$
\begin{aligned}
\sum_{N_{2} \delta=N \delta / N_{1}} f^{-1}(\delta) f\left(N_{2}\right) & =0, \text { if } \frac{N_{1}}{\delta} \neq N ; \\
& =1, \text { if } \frac{N_{1}}{\delta}=N .
\end{aligned}
$$

Thus the left side reduces to $f(M N)$. The right side is

$$
\sum \sum(-1)^{v} f\left(\frac{M}{t}\right) f\left(\frac{N}{\delta}\right) f^{-1}(\delta t),
$$

summed for all divisors $\delta$ of $N$, and all such divisors $t$ of $M$ as contain neither more nor less than the $\nu$ prime factors of $\delta$. It is clear that this is identical with

$$
\sum \sum f\left(\frac{M}{\delta_{1}}\right) f\left(\frac{N}{\delta_{2}}\right) f^{-1}\left(\delta_{1} \delta_{2}\right) C\left(\delta_{1}, \delta_{2}\right)
$$

$\delta$ being the special cardinal function defined above, and the summation extending over all the divisors $\delta_{1}$ of $M$, and $\delta_{2}$ of $N$. The identical equation of $f$, namely,

$$
f(M N)=\sum \sum f\left(\frac{M}{\delta_{1}}\right) f\left(\frac{N}{\delta_{2}}\right) f^{-1}\left(\delta_{1} \delta_{2}\right) C\left(\delta_{1}, \delta_{2}\right),
$$

is thus established.

4. The Identical Equation of the Quadratic Function. A multiplicative function $f$ may be called an integral quadratic function, or simply a quadratic function, if it is the composite of two linear functions. More generally, $f$ is an integral function of the rth degree, if it is the composite of $r$ linear functions. We shall now show that the identical equation of $f$ assumes a simple form when it is a quadratic function.

A fundamental property of the inverse function is: the inverse of the composite of any number of multiplicative functions of one argument is the composite of their inverses. This is easily proved, and is assumed in what follows.

Theorem 4. If $f(N)$ is an integral function of the rth degree, its inverse function $f^{-1}(N)$ vanishes whenever $N$ is divisible by an $(r+1)$ th power; also, if $N$ is the product of distinct primes,

$$
f^{-1}\left(N^{r}\right)=\{\mu(N)\}^{r} \lambda_{1}(N) \lambda_{2}(N) \cdots \lambda_{r}(N),
$$


where $f$ is the composite of the linear functions $\lambda_{1}, \lambda_{2}, \cdots, \lambda_{r}$, and $\mu(N)$ is Mertens' function.

For consider the theorem for the case $r=1$, that is, when $f$ is a linear function $\lambda(N)$. If $p$ is an arbitrary prime, we have from the definition of the inverse function

$$
\lambda(p)+\lambda^{-1}(p)=0 ; \lambda\left(p^{2}\right)+\lambda(p) \lambda^{-1}(p)+\lambda^{-1}\left(p^{2}\right)=0 .
$$

Hence $\lambda^{-1}(p)=-\lambda(p)$, and therefore $\lambda^{-1}(N)=\mu(N) \lambda(N)$, when $N$ is a product of distinct primes. Also, remembering that $\lambda$ is linear, we have $\lambda\left(p^{2}\right)=\lambda(p) \lambda(p)$, and therefore, from the second equation, $\lambda^{-1}\left(p^{2}\right)=0$. Hence $\lambda^{-1}(N)=0$ when $N$ has a square factor. The theorem is thus true for $r=1$.

Assume now the truth of the theorem for the value $r-1$ of $r$, and let $f$ be the composite of the linear functions $\lambda_{1}, \lambda_{2}, \cdots, \lambda_{r}$. Then $f^{-1} \lambda_{r}$ is the composite of the $r-1$ linearfunctions $\lambda_{1}, \lambda_{2}, \cdots, \lambda_{r-1}$. Hence, by virtue of our assumption, we may write

$$
\begin{aligned}
0=\left(f^{-1} \cdot \lambda_{r}\right)\left(p^{r}\right)= & f^{-1}\left(p^{r}\right)+f^{-1}\left(p^{r-1}\right) \lambda_{r}(p)+f^{-1}\left(p^{r-2}\right) \lambda_{r}\left(p^{2}\right)+\cdots, \\
0=\left(f^{-1} \cdot \lambda_{r}\right)\left(p^{r+1}\right)= & f^{-1}\left(p^{r+1}\right)+f^{-1}\left(p^{r}\right) \lambda_{r}(p)+\cdots \\
= & f^{-1}\left(p^{r+1}\right)+\lambda_{r}(p)\left\{f^{-1}\left(p^{r}\right)\right. \\
& \left.+f^{-1}\left(p^{r-1}\right) \lambda_{r}(p)+\cdots\right\} \\
= & f^{-1}\left(p^{r+1}\right)
\end{aligned}
$$

since $\lambda_{r}$ is linear. Hence $f^{-1}(N)=0$ if $N$ is divisible by an $(r+1)$ th power. Again, for the second part of the theorem, we have, by virtue of our assumption,

$(-1)^{r-1} \lambda_{1}(p) \lambda_{2}(p) \cdots \lambda_{r-1}(p)=\left(f^{-1} \cdot \lambda_{r}\right)\left(p^{r-1}\right)$

Hence

$$
=f^{-1}\left(p^{r-1}\right)+f^{-1}\left(p^{r-2}\right) \lambda_{r}(p)+\cdots \cdot
$$

$$
\begin{aligned}
0 & =\left(f^{-1} \cdot \lambda_{r}\right)\left(p^{r}\right) \\
& =f^{-1}\left(p^{r}\right)+\lambda_{r}(p)\left\{f^{-1}\left(p^{r-1}\right)+\lambda_{r}(p) f^{-1}\left(p^{r-2}\right)+\cdots\right\} \\
& =f^{-1}\left(p^{r}\right)+(-1)^{r-1} \lambda_{1}(p) \lambda_{2}(p) \cdots \lambda_{r}(p)
\end{aligned}
$$

or

$$
\begin{aligned}
& f^{-1}\left(p^{r}\right)=(-1)^{r} \lambda_{1}(p) \lambda_{2}(p) \cdots \lambda_{r}(p) ; \\
& f^{-1}\left(N^{r}\right)=\{\mu(N)\}^{r} \lambda_{1}(N) \cdots \lambda_{r}(N),
\end{aligned}
$$


$N$ being a product of distinct primes. Thus the theorem is true for the next higher value of $r$. Since it has been seen to be true for $r=1$, the induction is complete.

THEOREM 5. If $f$ is the composite of the two linear functions $\lambda_{1}(N), \lambda_{2}(N)$, its identical equation takes the form

$$
f(M N)=\sum f\left(\frac{M}{\delta}\right) f\left(\frac{N}{\delta}\right) \lambda_{1}(\delta) \lambda_{2}(\delta) \nu(\delta),
$$

where the summation is now for all common divisors $\delta$ of $M, N$.

Proof. The identical equation of $f$ may be written in the form

$$
f(M N)=\sum f\left(\frac{M}{\delta_{1}}\right) f\left(\frac{N}{\delta_{2}}\right) \cdot(-1)^{v} f^{-1}\left(\delta_{1} \delta_{2}\right),
$$

summed for pairs of divisors $\delta_{1}, \delta_{2}$ of $M, N$, which contain the same distinct prime factors, $\nu$ in number. Now if $f$ is a quadratic function, $f^{-1}\left(\delta_{1} \delta_{2}\right)$ vanishes when $\delta_{1} \delta_{2}$ contains a cubed factor and therefore (since $\delta_{1}, \delta_{2}$ contain the same distinct prime factors) when either $\delta_{1}$ or $\delta_{2}$ contains a squared factor. It follows that the only non-zero terms in the above sum are those for which

Hence

$$
\delta_{1}=\delta_{2}=\delta=\text { a product of distinct primes. }
$$

$$
f(M N)=\sum f\left(\frac{M}{\delta}\right) f\left(\frac{N}{\delta}\right) \cdot(-1)^{v} f^{-1}\left(\delta^{2}\right),
$$

summed for common divisors $\delta$ of $M, N$. If we use Theorem 4 , this becomes

$$
\begin{aligned}
f(M N) & =\sum f\left(\frac{M}{\delta}\right) f\left(\frac{N}{\delta}\right) f^{-1}\left(\delta^{2}\right) \mu(\delta) \\
& =\sum f\left(\frac{M}{\delta}\right) f\left(\frac{N}{\delta}\right) \lambda_{1}(\delta) \lambda_{2}(\delta) \mu(\delta) .
\end{aligned}
$$

As special cases of this theorem, may be mentioned the identical equations of the following functions:

(1) $\sigma_{a}(N)$ (= the sum of the $a$ th powers of the divisors of $N$ ). This is a quadratic function, being the composite of the two linear functions $\lambda_{1}(N)=N^{a}$, and $\lambda_{2}(N)=1$. Hence 


$$
\sigma_{a}(M N)=\sum \sigma_{a}\left(\frac{M}{\delta}\right) \sigma_{a}\left(\frac{N}{\delta}\right) \delta^{a} \mu(\delta),
$$

summed for common divisors $\delta$.

(2) The function $4 R(N)$ which is equal to the number of representations of $N$ as a sum of two squares. It is known that $R(N)$ is also equal to the excess of the number of divisors of $N$ of the form $4 n+1$ over the number of those of the form $4 n-1$. Hence $R(N)$ is a quadratic function, being the composite of the linear functions $\lambda_{1}(N), \lambda_{2}(N)$ defined by the quadratic residue symbol

Hence

$$
\lambda_{2}(N)=1 ; \lambda_{1}\left(2^{n}\right)=0 ; \lambda_{1}(N)=\left(\frac{-1}{N}\right),
$$

$$
R(M N)=\sum R\left(\frac{M}{\delta}\right) R\left(\frac{N}{\delta}\right)\left(\frac{-1}{\delta}\right) \mu(\delta),
$$

summed for common divisors $\delta$ of $M, N$.

Madras University, INDIA 